\title{
Anatomical Importance of Inner Ligamentum Flavum Parameters for Successful Endoscopic Lumbar Decompression Surgery
}

\author{
Ji Yeon Kim², Hyeun Sung Kim², Pang Hung $\mathrm{Wu}^{2}$, Il-Tae Jang ${ }^{1}$ \\ ${ }^{1}$ Department of Neurosurgery, Nanoori Gangnam Hospital, Seoul, Spine Surgery, Seoul, Republic of Korea \\ ${ }^{2}$ Department of Orthopaedic Surgery, National University Health System, Jurong Health Campus, Singapore
}

Corresponding Author:

Hyeun Sung Kim, MD, PhD

Department of Neurosurgery, Nanoori

Hospital Gangnam, Seoul 731, Eonju-ro,

Gangnam-gu, Seoul, Republic of Korea

Tel: $+82-2-6003-9767$

Fax: +82-2-3445-9755

Email: neurospinekim@gmail.com

Received: October 06, 2020

Revised: December 20, 2020

Accepted: January 10, 2021
Objective: Predicting the gross anatomy of the inner ligament flavum and surrounding interlaminar window is essential for sufficient neural decompression because endoscopic spine surgery is performed in a limited, narrow surgical corridor. This article aims to analyze the anatomical data of inner LF and interlaminar window on the 3D lumbar computed tomography (CT) for easy application to the endoscopic decompression surgery. Methods: We measured nine parameters indicating the contour of inner LF and interlaminar window on 3D CT from 100 patients who were diagnosed with lumbar spinal stenosis or disc herniation. Inner LF angle, inner LF distance, inner LF volume, and inner foraminal ligament distance for inner LF contour; height, width, and lateral corner angle of interlaminar window contour were measured in five age groups consisted of twenty individuals from the 30s to the 70s. We then compared two age groups (ages 30-49 years, $n=40$; ages $60-79$ years, $n=40$ ). Results: In the old age group, the interlaminar window was changed to a smaller triangle shape representing decreased width and height and increased lateral corner angle. Inner LF volume, inner LF angle, inner LF distance were also reduced in the old age group. But these parameters have variations in levels with a significant difference. Uniportal endoscopic docking point has moved according to changed interlaminar window features more caudal and lateral direction. Inner LF angle could have used to decide the approach angle for endoscopic contralateral foraminotomy. Conclusion: We found the changing features of these structures correlated with age and levels to help with endoscopic decompression surgery has a limited surgical corridor. The present study results may help the endoscopic surgeons decide the endoscopic docking site, the extent of bone drilling at the medial facet joint part, and the approach angle for contralateral lumbar foraminotomy.

Key Words: Ligamentum flavum, Lumbar spine, Endoscopic spine surgery, Spinal stenosis

\section{INTRODUCTION}

Ligamentum flavum (LF) is a component of the posterior ligamentous complex of the spine, extending between the arcus vertebrae. It originates from the superior-most aspect of the inferior lamina and extends onto the anteroinferior surface of the superior lamina. This bifid ligament consists of a superficial layer and a deep layer whose fibers run in opposing directions ${ }^{2,10,11)}$. Hypertrophy of the LF is a significant cause of lumbar central spinal stenosis. Previous studies have indicated that LF shows a loss of elasticity with aging that may cause LF to fold into the spinal canal, which may further narrow the canal ${ }^{11,12)}$. Thus, the spatial relationship of LF with its surrounding structures could potentially guide surgeons in planning their surgical strategy ${ }^{9,10)}$.

During endoscopic decompression surgery, most procedures are performed while the scope is inside the spinal canal, so that the surgical corridor is limited and very narrow. Under these circumstances, the exact prediction of the inner LF contour within the endoscopic view is crucial for sufficient neural decompression. Although the general information of lumbar LF contour and its aging changes have been known to the spinal surgeons. Previous anatomical studies have focused only on magnetic resonance imaging (MRI) section images or cadaver dissection, and not on spatial relationships seen in gross anatomy ${ }^{1,2,13)}$.

Hence, we set the parameters on the 3D computed tomography (CT) image for easy application of measured values and predict the extent of decompression before and during the 
endoscopic surgery. The interlaminar window boundaries and correlated LF contour were decided to essential measuring parameters, because these could directly affect the endoscope procedures during the spinal canal decompression. We then compared values between the middle-age group (patients in the 30s and 40s) and the old-age group (patients in the 60s and 70s) to confirm the changing features with age.

This article aims to analyze the anatomical data of inner LF and interlaminar window on the 3D lumbar CT for easy application to the endoscopic decompression surgery in patients with lumbar spinal stenosis and disc herniation.

\section{MATERIALS AND METHODS}

\section{Study Patients}

This article is a retrospective review of 100 patients diagnosed with lumbar disc herniation or lumbar spinal stenosis after performing the 3D lumbar CT and MRI between January 2018 and December 2019 at a single center (Gangnam Nanoori Hospital, Seoul, Korea). The patients were randomly selected, regardless of their symptoms and treatments, including conservative or surgical procedures. The included patients have the same gender distribution ( $M: F=50: 50)$, and five age groups consisted of twenty individuals from the 30 s to the 70 s. For confirming the change of values with age, patients were divided into a middle-aged group (ages 30-49 years, $\mathrm{n}=40$ ) and an old-aged group (ages 6079 years, $n=40$ ), then compared the values to assess the significant difference between two-age groups at the consecutive lumbar levels. We excluded patients due to the followings: previous laminectomy or fusion operation, ankylosing spondylitis, fused vertebral body, lamina bifida, scoliosis with Cobb's angle> $10^{\circ}$, or vertebral body compression fracture. The Ethics Commi-

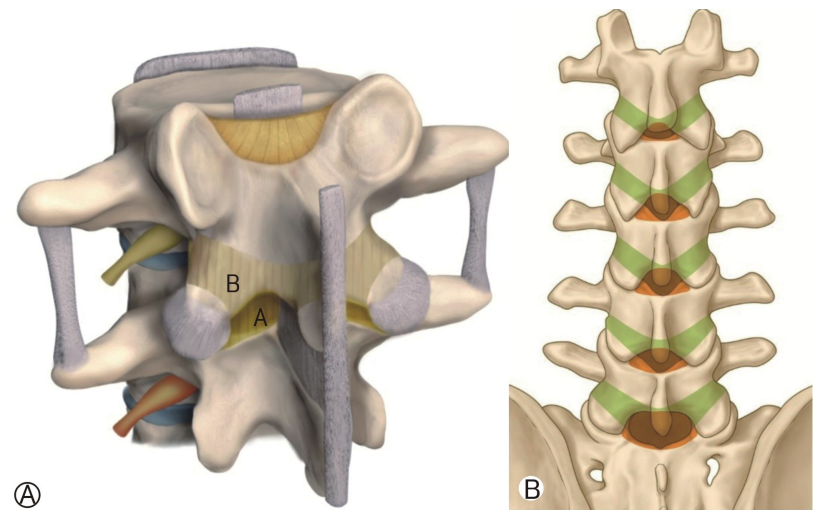

Figure 1. (1) Anatomical configuration of ligamentum flavum. (A): Outer layer of ligamentum flavum, (B): Inner layer of ligamentum flavum. This bifid ligament originates from the most superior aspect of the inferior lamina and inserts onto the anteroinferior surface of the superior lamina. Still, the inner layer has a broader extension to more laterally attaches to the inferior-lateral border of the upperlevel pedicle and highest tip of the superior articular process. (2) Illustration of ligamentum flavum contour on the gross anatomy. Green boomerang shape. ttee of Nanoori Hospital approved this study.

\section{Imaging Analysis}

One junior professional neurosurgeon measured the parameters twice consecutively on the CT, and the average values were used in this article. Bilateral parameters were measured, and average values were used due to the anatomical asymmetry. The images in this study were obtained using a CT Machine (Supria, WCT-400-140, HITACHI, Japan). All measurements were taken on 3D reconstructed lumbar CT images using an Infinitt PACS M6 Version (Infinitt Healthcare Co., Seoul, Korea). We measured nine values to characterize the anatomical features of lumbar level laminas and the LF, two values for the outer lamina, three values for the interlaminar window, and four values for the inner LF. Most of the measurements were made by drawing a line in AP 3D images. For some obscured regions, we used a little rotated 3D images (internal foraminal ligament distance), as described below. After measuring the length and angle from both the left and right sides, we calculated the mean values to reduce deviations.

\section{Three Parameters for Interlaminar Window Bounda- ries (Figure 1)}

Sagittal interlaminar distance (SILD): The vertical linear distance between the spinolaminar junction and the upper margin of the lower lamina.

Transverse interlaminar distance (TILD): The transverse linear distance between the midline and the most lateral margin of the interlaminar space.

Faceto-midline angle (FMA): The angle between a line along the lower margin of the lamina and a line connecting the lower end of both inferior articular processes.

\section{Two Parameters for Outer Laminar Contour (Figure 2)}

Sagittal laminae length (SLL): The vertical linear distance bet-

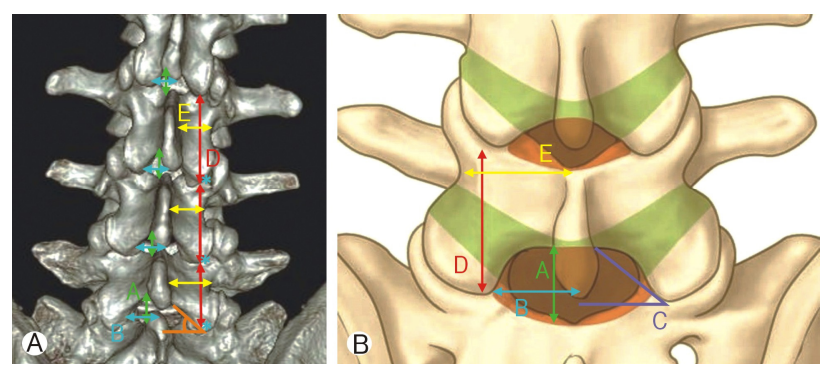

Figure 2. Measurement of the interlaminar window boundaries and outer lamina contour. (1) The actual measurement of parameters on the 3D lumbar CT image. (2) Illustration of parameters on the lumbar spine model. (A): sagittal interlaminar distance (SILD), (B): transverse interlaminar distance (TILD), (C): faceto-midline angle (FMA), (D): sagittal laminae length (SLL), (E): transverse laminae length (TLL). *: Caudal point of facet joint, distance $(\mathrm{mm})$, angle $\left({ }^{\circ}\right)$. 
ween the inferior end of the upper-level facet joint and the caudal end of the index level inferior articular process. This parameter is representing the gross anatomical sagittal laminae length, not the actual length.
Transverse laminae length (TLL): The transverse linear distance between the lateral border of the isthmus and the midline of the spinous process.
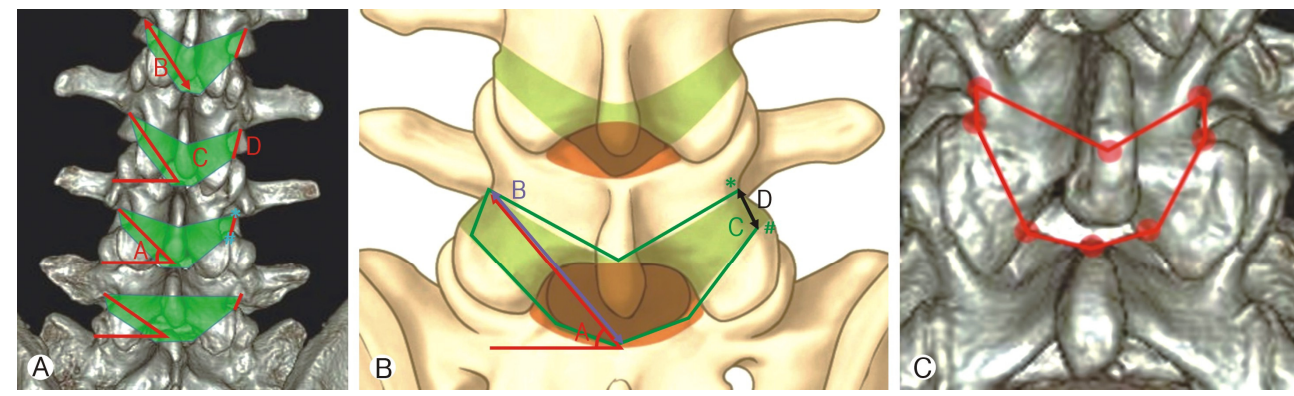

Figure 3. Measurement of the inner ligamentum flavum contour. (1) The actual measurement of parameters on 3D lumbar CT image. (2) Illustration of parameters on the lumbar spine model. (3) The volume of the inner ligamentum flavum was measured by connecting eight points. (A): Inner ligamentum flavum angle (ILFA), (B): Inner ligamentum flavum distance (ILFD), (C): Inner ligamentum flavum volume (ILFV), D: Inner foraminal ligament distance (IFLD). *: Lateral border of pedicle, \#: Cranial point of facet joint. Distance $(\mathrm{mm})$, Angle $\left({ }^{\circ}\right)$, Volume $\left(\mathrm{mm}^{2}\right)$.

Table 1. Summary of the mean values of outer lamina and outer ligamentum flavum parameters by age groups

\begin{tabular}{|c|c|c|c|c|c|c|}
\hline Variable & Age & L1-L2 & L2-L3 & L3-L4 & L4-L5 & L5-S1 \\
\hline \multirow{6}{*}{ SILD (mm) } & 30-39 & $23.3 \pm 4.9$ & $23.6 \pm 4.8$ & $22.8 \pm 4.7$ & $21.4 \pm 4.9$ & $24.3 \pm 5.6$ \\
\hline & $40-49$ & $23.0 \pm 4.9$ & $23.3 \pm 4.5$ & $21.8 \pm 4.0$ & $21.4 \pm 4.2$ & $27.0 \pm 5.0$ \\
\hline & $50-59$ & $22.9 \pm 4.8$ & $22.2 \pm 4.3$ & $19.4 \pm 4.3$ & $18.8 \pm 4.5$ & $23.8 \pm 3.1$ \\
\hline & $60-69$ & $19.7 \pm 4.7$ & $20.7 \pm 4.9$ & $19.0 \pm 5.6$ & $17.2 \pm 5.6$ & $22.1 \pm 4.2$ \\
\hline & $70-79$ & $19.1 \pm 3.1$ & $19.4 \pm 3.2$ & $18.9 \pm 4.0$ & $18.2 \pm 4.1$ & $22.8 \pm 5.7$ \\
\hline & Total & $21.6 \pm 4.9$ & $21.8 \pm 4.6$ & $20.4 \pm 4.8$ & $19.4 \pm 5.0$ & $24.0 \pm 5.1$ \\
\hline \multirow{6}{*}{ TILD (mm) } & 30-39 & $14.3 \pm 3.3$ & $16.3 \pm 2.9$ & $17.9 \pm 3.1$ & $21.4 \pm 2.8$ & $27.8 \pm 4.5$ \\
\hline & $40-49$ & $14.3 \pm 2.7$ & $15.9 \pm 3.1$ & $18.1 \pm 4.4$ & $21.7 \pm 4.3$ & $30.1 \pm 5.7$ \\
\hline & $50-59$ & $14.0 \pm 3.6$ & $15.1 \pm 3.6$ & $16.6 \pm 3.2$ & $19.1 \pm 3.7$ & $24.8 \pm 4.2$ \\
\hline & $60-69$ & $13.7 \pm 2.6$ & $15.2 \pm 2.7$ & $16.8 \pm 3.5$ & $20.4 \pm 4.6$ & $26.1 \pm 4.4$ \\
\hline & $70-79$ & $13.1 \pm 2.9$ & $14.3 \pm 2.9$ & $15.6 \pm 3.3$ & $19.8 \pm 3.7$ & $25.9 \pm 4.0$ \\
\hline & Total & $13.9 \pm 3.1$ & $15.3 \pm 3.1$ & $17.0 \pm 3.7$ & $20.5 \pm 4.0$ & $26.9 \pm 5.0$ \\
\hline \multirow{6}{*}{ FMA $\left({ }^{\circ}\right)$} & $30-39$ & $51.4 \pm 6.6$ & $49.5 \pm 7.4$ & $46.8 \pm 6.0$ & $40.8 \pm 5.4$ & $33.4 \pm 6.3$ \\
\hline & $40-49$ & $49.4 \pm 8.5$ & $48.2 \pm 5.2$ & $43.9 \pm 6.9$ & $38.4 \pm 8.9$ & $29.9 \pm 5.4$ \\
\hline & $50-59$ & $46.1 \pm 8.2$ & $45.4 \pm 5.6$ & $44.3 \pm 4.6$ & $38.0 \pm 5.4$ & $30.5 \pm 6.3$ \\
\hline & $60-69$ & $46.5 \pm 6.3$ & $46.4 \pm 5.9$ & $42.9 \pm 4.7$ & $38.4 \pm 7.0$ & $31.3 \pm 6.3$ \\
\hline & $70-79$ & $53.7 \pm 8.1$ & $52.7 \pm 6.8$ & $49.9 \pm 5.9$ & $46.4 \pm 6.7$ & $35.0 \pm 7.2$ \\
\hline & Total & $49.4 \pm 8.1$ & $48.4 \pm 6.7$ & $45.6 \pm 6.2$ & $40.4 \pm 7.5$ & $32.0 \pm 6.6$ \\
\hline \multirow{6}{*}{$\mathrm{SLL}(\mathrm{mm})$} & $30-39$ & $64.4 \pm 7.2$ & $68.9 \pm 7.3$ & $67.0 \pm 5.6$ & $62.1 \pm 5.4$ & $53.3 \pm 6.4$ \\
\hline & $40-49$ & $64.8 \pm 5.5$ & $68.6 \pm 5.3$ & $66.5 \pm 5.9$ & $59.4 \pm 4.9$ & $49.9 \pm 6.0$ \\
\hline & $50-59$ & $64.6 \pm 5.5$ & $69.1 \pm 5.6$ & $68.2 \pm 6.0$ & $62.0 \pm 6.5$ & $51.2 \pm 4.8$ \\
\hline & $60-69$ & $63.1 \pm 7.2$ & $68.4 \pm 6.4$ & $65.7 \pm 5.4$ & $57.3 \pm 5.9$ & $48.3 \pm 5.2$ \\
\hline & $70-79$ & $62.1 \pm 6.3$ & $66.2 \pm 6.8$ & $65.1 \pm 6.1$ & $54.8 \pm 6.4$ & $47.2 \pm 7.6$ \\
\hline & Total & $63.8 \pm 6.5$ & $68.2 \pm 6.4$ & $66.5 \pm 5.9$ & $59.1 \pm 6.5$ & $50.0 \pm 6.4$ \\
\hline \multirow{6}{*}{$\mathrm{TLL}(\mathrm{mm})$} & $30-39$ & $21.8 \pm 4.4$ & $24.6 \pm 3.7$ & $28.2 \pm 3.6$ & $34.2 \pm 4.2$ & $41.5 \pm 5.1$ \\
\hline & $40-49$ & $19.9 \pm 3.0$ & $24.1 \pm 3.4$ & $28.0 \pm 3.2$ & $32.5 \pm 3.8$ & $40.5 \pm 3.5$ \\
\hline & $50-59$ & $20.0 \pm 3.2$ & $23.3 \pm 4.2$ & $25.9 \pm 4.4$ & $30.8 \pm 5.5$ & $36.5 \pm 6.3$ \\
\hline & $60-69$ & $20.0 \pm 3.3$ & $23.3 \pm 3.2$ & $27.3 \pm 3.8$ & $32.2 \pm 5.7$ & 39.56 .7 \\
\hline & $70-79$ & $20.1 \pm 3.1$ & $22.8 \pm 3.6$ & $26.4 \pm 3.9$ & $30.9 \pm 4.4$ & $38.9 \pm 4.5$ \\
\hline & Total & $20.4 \pm 3.5$ & $23.6 \pm 3.7$ & $27.2 \pm 3.9$ & $32.1 \pm 4.9$ & $39.4 \pm 5.6$ \\
\hline
\end{tabular}

SILD: sagittal interlaminar distance, TILD: transverse interlaminar distance, FMA: faceto-midline angle, SLL: sagittal laminae length, TLL: transverse laminae length. Values are presented as the mean \pm standard deviation. 


\section{Four Parameters for Inner LF Contours (Figure 3)}

Inner ligamentum flavum angle (ILFA): The angle between a line parallel to the vertebral body and a line connecting the inferior-lateral end of the upper pedicle and the upper end of the lower lamina at the midline.

Inner ligamentum flavum distance (ILFD): The linear distance between the inferior-lateral end of the upper pedicle and the upper end of the lower lamina at the midline.

Inner ligamentum flavum volume (ILFV): The volume of the inner LF contour was measured by connecting eight points. These eight points were the bilateral inferior-lateral end of the upper pedicle, bilateral cranial point of facet joints, the bilateral junction of the superior articular process and cranial margin of the lamina and the point, midline superior-end point of the lower lamina, and $8 \mathrm{~mm}$ above the imaginary line that meets the bilateral spinolaminar junctions (Figure 3-3).

Inner foraminal ligament distance (IFLD): The linear distance between the inferior-lateral end of the upper pedicle and the facet joint's cranial point was measured.

\section{Statistical Analysis}

Statistical analyses were performed using PASW Statistics ver. 18.0 (SPSS Inc., Chicago, IL, USA). The mean values of each parameter were calculated at consecutive lumbar levels for five age groups. Statistical comparison of all parameter values at the successive levels between two age groups was done using the independent $t$-test. A p-value $<0.05$ was considered to indicate a statistically significant difference.

\section{RESULTS}

We measured the mean $\mathrm{S} L$ and $T \amalg$ as outer laminar contours; SILD, TILD, and FMA as interlaminar window boundaries; and ILFA, ILFD, ILFV, and IFLD as inner LF contours at all lumbar levels by age group. We analyzed the values focusing on accuracy and anatomical changes according to age and level(Figures 1, 2, 3).

The SILD represented the height of the interlaminar window. The mean SILD decreased with age at all levels; meanwhile, according to the levels, it was higher at the L1-L2, L2-L3 level than at the L3-L4, L4-L5 level, and highest at the L5-S1 level through all age group. The TILD means the unilateral width of the interlaminar window. The TILD was gradually increasing from the L1-2 to the L5-S1 level in all age groups. At the L1-L2 and L2-L3 level, the TILD decreases progressively with age; however, at the L3-L4 level and below, it showed many variations without definite direction (Table 1).

The FMA represents the gross shape of the interlaminar win-

Table 2. Summary of the mean values of inner ligamentum flavum parameters by age groups

\begin{tabular}{|c|c|c|c|c|c|c|}
\hline Variable & Age & L1-L2 & L2-L3 & L3-L4 & L4-L5 & L5-S1 \\
\hline \multirow{6}{*}{ ILFA $\left(^{\circ}\right)$} & $30-39$ & $57.1 \pm 3.3$ & $54.7 \pm 3.6$ & $51.4 \pm 4.3$ & $43.7 \pm 6.0$ & $34.1 \pm 7.0$ \\
\hline & $40-49$ & $57.6 \pm 4.7$ & $55.2 \pm 3.4$ & $50.1 \pm 4.2$ & $42.2 \pm 4.2$ & $31.6 \pm 5.4$ \\
\hline & $50-59$ & $57.1 \pm 2.8$ & $54.4 \pm 4.1$ & $49.1 \pm 3.6$ & $41.6 \pm 4.9$ & $31.1 \pm 5.4$ \\
\hline & $60-69$ & $56.3 \pm 3.3$ & $53.7 \pm 3.3$ & $49.0 \pm 6.3$ & $40.1 \pm 7.1$ & $31.2 \pm 5.6$ \\
\hline & $70-79$ & $53.2 \pm 4.0$ & $50.0 \pm 3.3$ & $46.4 \pm 5.2$ & $38.8 \pm 6.8$ & $31.1 \pm 6.0$ \\
\hline & Total & $56.2 \pm 4.0$ & $53.6 \pm 4.0$ & $49.2 \pm 5.1$ & $41.3 \pm 6.1$ & $31.8 \pm 6.0$ \\
\hline \multirow{6}{*}{ ILFD (mm) } & 30-39 & $67.2 \pm 4.6$ & $69.7 \pm 4.4$ & $68.2 \pm 3.3$ & $61.7 \pm 5.2$ & $58.5 \pm 6.6$ \\
\hline & $40-49$ & $67.3 \pm 5.1$ & $69.3 \pm 5.6$ & $67.9 \pm 5.1$ & $61.3 \pm 5.3$ & $62.1 \pm 7.4$ \\
\hline & $50-59$ & $64.8 \pm 4.4$ & $66.3 \pm 4.8$ & $63.6 \pm 5.1$ & $59.3 \pm 6.0$ & $56.1 \pm 6.5$ \\
\hline & $60-69$ & $65.6 \pm 5.7$ & $67.4 \pm 4.6$ & $64.5 \pm 5.3$ & $57.5 \pm 5.1$ & $58.7 \pm 7.5$ \\
\hline & $70-79$ & $60.7 \pm 6.3$ & $63.5 \pm 6.5$ & $61.0 \pm 6.7$ & $58.3 \pm 6.6$ & $58.8 \pm 6.9$ \\
\hline & Total & $65.1 \pm 5.8$ & $67.2 \pm 5.7$ & $65.0 \pm 5.9$ & $59.6 \pm 5.9$ & $58.8 \pm 7.2$ \\
\hline \multirow{6}{*}{ ILFV $\left(\mathrm{mm}^{2}\right)$} & 30-39 & $2176 \pm 363$ & $2411 \pm 354$ & $2578 \pm 380$ & $2821 \pm 395$ & $3147 \pm 468$ \\
\hline & $40-49$ & $2214 \pm 290$ & $2468 \pm 332$ & $2639 \pm 368$ & $2825 \pm 503$ & $3365 \pm 526$ \\
\hline & $50-59$ & $1885 \pm 297$ & $2103 \pm 305$ & $2209 \pm 344$ & $2346 \pm 474$ & $2818 \pm 409$ \\
\hline & $60-69$ & $1833 \pm 419$ & $1889 \pm 480$ & $1930 \pm 534$ & $2336 \pm 628$ & $2835 \pm 556$ \\
\hline & $70-79$ & $1712 \pm 358$ & $1914 \pm 341$ & $1982 \pm 335$ & $2283 \pm 353$ & $2798 \pm 460$ \\
\hline & Total & $1964 \pm 401$ & $2158 \pm 433$ & $2268 \pm 474$ & $2522 \pm 540$ & $2993 \pm 536$ \\
\hline \multirow{6}{*}{ IFLD (mm) } & 30-39 & $23.8 \pm 3.7$ & $25.6 \pm 3.4$ & $22.4 \pm 3.0$ & $16.8 \pm 2.8$ & $11.2 \pm 2.8$ \\
\hline & $40-49$ & $23.3 \pm 2.7$ & $24.5 \pm 4.6$ & $21.9 \pm 3.7$ & $16.0 \pm 3.6$ & $11.3 \pm 1.7$ \\
\hline & $50-59$ & $21.6 \pm 3.2$ & $21.3 \pm 2.7$ & $20.1 \pm 3.5$ & $14.7 \pm 2.6$ & $9.6 \pm 0.9$ \\
\hline & $60-69$ & $19.9 \pm 3.8$ & $20.7 \pm 3.8$ & $19.0 \pm 3.1$ & $14.1 \pm 3.5$ & $9.3 \pm 2.1$ \\
\hline & $70-79$ & $17.7 \pm 2.9$ & $16.9 \pm 2.6$ & $14.9 \pm 3.0$ & $11.5 \pm 2.1$ & $8.1 \pm 1.6$ \\
\hline & Total & $21.2 \pm 4.0$ & $21.8 \pm 4.8$ & $19.7 \pm 4.2$ & $14.6 \pm 3.5$ & $9.9 \pm 2.2$ \\
\hline
\end{tabular}

ILFA: inner ligamentum flavum angle, ILFD: inner ligamentum flavum distance, ILEF: inner ligamentum flavum volume, IFLD: inner foraminal ligament distance. Values are presented as the meantstandard deviation. 
dow, such as the lower FAM value, which was shown a more broad-based triangular contour. As the levels down, the FMA gradually decreased in all levels. Meanwhile, it showed a decreasing tendency with age, but highest in the 70s at all lumbar levels (Table 1).

The SLL represents the gross anatomical sagittal laminae length on the $3 \mathrm{D}$ lumbar $\mathrm{CT}$, which could be changed by the age and degenerative process. The SLL showed the height value at the L2-L3 level, thereafter, the values gradually decreased as the levels down in all age groups. However, there was no specific direction of changes with age at all levels (Table 1).

The TLL means the lamina width at the isthmus level, which describes the lamina shape according to the lumbar levels. The TLL increased gradually as the levels down and showed the highest increment between the L4-L5 and L5-S1 levels. There were variations in changes without a consistent direction with age in all levels (Table 1).

The ILFA represents the maximal endoscopic approach angle from the midline to the upper end of the contralateral foramen for resolving contralateral foraminal pathology. The ILFA decreased as the levels down in all age groups, and higher decrements were showed at lower lumbar levels, the L4-L5, and L5-S1 levels. Furthermore, it was gradually decreased with age in all lumbar levels (Table 2).

The ILFD expresses the length of sublaminar decompression for contralateral lateral recess and foraminal decompression from the midline to the upper end of the contralateral foramen. The ILFD showed the height value at the L2-L3 level; after that, the values gradually decreased as the levels down in all age groups, and the marked lower values were revealed at the L4-L5 and L5-S1 level. However, there was no specific direction of changes with age (Table 2).
The ILFV is closely correlated with anatomical features of the vertebral level and its degenerative changes. The ILFV showed the consistent direction of changes according to the age and the lumbar levels. The ILFV increased as the levels down, and height increment was found between the L4-L5 and L5-S1 levels in all age groups. Furthermore, the values gradually decreased with age in all lumbar levels (Table 2).

The IFLD expresses the inter-foraminal distance correlated with facet joint spatial orientation, which is affected by the anatomical features of each lumbar level, disc height loss, and facet joint hypertrophy. The IFLD was the highest at the L2-3 level; after that, the value gradually decreased as the lumbar levels down in all age groups, and markedly low values were showed at the L4-L5, L5-S1 levels. These results could be described by the anatomical features of the lower lumbar spine, such as large facet joint and increased segmental lordosis. According to the age, the IFLD gradually decreased, and marked decrement was found at the L5-S1 level (Table 2).

We analyzed the mean SILD, TILD, FMA, SLL, TLL, ILFA, ILFD, ILFV, and IFLD values at consecutive levels for two age groups, then, compared them to find significant difference. The mean SILD, TILD, SLL, TLL, ILFA, ILFD, ILFV, and IFLD values were lower in the old-age group than in the middle-age group at all levels. Meanwhile, the mean FMA values were higher in the old-age group than in the middle-age group at the L2-L3, L3-L4, L4-L5, and L5-S1 levels (Tables 3, 4) (Figures. 4, 5). There were significant differences in the SILD at all levels $(p=0.000$ for $L 1-L 2$, $p=0.001$ for $L 2-L 3, p=0.002$ for $L 3-L 4, p=0.001$ for $L 4-L 5, p=0.009$ for $L 5-1)$; in the TILD, at $L 3-L 4(p=0.033)$ and $L 5-S 1 \quad(p=0.008)$; however, there was no significant difference in the FMA at all levels. The SLL showed a significant difference at L4-L5 $(p=$ $0.001)$ and L5-S1 ( $p=0.011)$, but the TLL did not show any signi-

Table 3. Comparison of outer lamina and outer ligamentum flavum parameters between two age groups

\begin{tabular}{|c|c|c|c|c|c|c|c|c|c|}
\hline \multirow{2}{*}{ Level } & \multicolumn{3}{|c|}{ SILD (mm) } & \multicolumn{3}{|c|}{ TILD (mm) } & \multicolumn{3}{|c|}{ FMA ( $\left(^{\circ}\right)$} \\
\hline & Middle & Old & $\mathrm{p}$-value & Middle & Old & p-value & Middle & Old & $\mathrm{p}$-value \\
\hline L1-L2 & $23.1 \pm 4.9$ & $19.4 \pm 4.0$ & $\mathrm{p}=0.000^{*}$ & $14.3 \pm 3.0$ & $13.4 \pm 2.8$ & $p=0.192$ & $50.4 \pm 7.7$ & $50.1 \pm 8.1$ & $p=0.867$ \\
\hline L2-L3 & $23.4 \pm 4.6$ & $20.0 \pm 4.2$ & $p=0.001^{*}$ & $16.1 \pm 3.0$ & $14.7 \pm 2.9$ & $p=0.05$ & $48.8 \pm 6.5$ & $49.6 \pm 7.1$ & $p=0.637$ \\
\hline L3-L4 & $22.3 \pm 4.4$ & $19.0 \pm 4.9$ & $p=0.002^{*}$ & $18.0 \pm 3.8$ & $16.2 \pm 3.4$ & $p=0.033^{*}$ & $45.3 \pm 6.6$ & $46.4 \pm 6.4$ & $p=0.475$ \\
\hline L4-L5 & $21.4 \pm 4.6$ & $17.7 \pm 5.0$ & $p=0.001^{*}$ & $21.5 \pm 3.6$ & $20.1 \pm 4.2$ & $p=0.117$ & $39.6 \pm 7.4$ & $42.4 \pm 7.9$ & $p=0.121$ \\
\hline L5-S1 & $25.6 \pm 5.5$ & $22.4 \pm 5.0$ & $p=0.009^{*}$ & $29.0 \pm 5.3$ & $26.0 \pm 4.2$ & $p=0.008^{*}$ & $31.6 \pm 6.1$ & $33.1 \pm 7.0$ & $p=0.315$ \\
\hline \multirow{2}{*}{ Level } & \multicolumn{3}{|c|}{$\mathrm{SLL}(\mathrm{mm})$} & \multicolumn{3}{|c|}{ TLL (mm) } & & & \\
\hline & Middle & Old & $\mathrm{p}$-value & Middle & Old & $\mathrm{p}$-value & & & \\
\hline L1-L2 & $64.6 \pm 6.4$ & $62.6 \pm 6.8$ & $p=0.182$ & $20.8 \pm 3.9$ & $20.1 \pm 3.2$ & $p=0.357$ & & & \\
\hline L2-L3 & $68.7 \pm 6.4$ & $67.3 \pm 6.7$ & $p=0.341$ & $24.3 \pm 3.5$ & $23.1 \pm 3.4$ & $p=0.134$ & & & \\
\hline L3-L4 & $66.7 \pm 5.8$ & $65.4 \pm 5.8$ & $p=0.31$ & $28.1 \pm 3.4$ & $26.8 \pm 3.9$ & $p=0.13$ & & & \\
\hline L4-L5 & $60.8 \pm 5.3$ & $56.1 \pm 6.3$ & $p=0.001^{*}$ & $33.0 \pm 4.1$ & $31.5 \pm 5.1$ & $p=0.091$ & & & \\
\hline L5-S1 & $51.6 \pm 6.4$ & $47.8 \pm 6.5$ & $p=0.011^{*}$ & $41.0 \pm 4.4$ & $39.2 \pm 5.7$ & $p=0.128$ & & & \\
\hline
\end{tabular}

SILD: sagittal interlaminar distance, TILD: transverse interlaminar distance, FMA: faceto-midline angle, SLL: sagittal laminae length, TLL: transverse laminae length. Middle: ages 30-49 years; Old: ages 60-79 years. Values are presented as the mean \pm standard deviation.

*Statistically significant difference, $p<0.05$. 
Table 4. Comparison of inner ligamentum flavum parameters between two age groups.

\begin{tabular}{|c|c|c|c|c|c|c|}
\hline \multirow{2}{*}{ Level } & \multicolumn{3}{|c|}{ ILFA ( $\left.{ }^{\circ}\right)$} & \multicolumn{3}{|c|}{ ILFD (mm) } \\
\hline & Middle & Old & $\mathrm{p}$-value & Middle & Old & $\mathrm{p}$-value \\
\hline L1-L2 & $57.3 \pm 4.1$ & $54.7 \pm 4.0$ & $p=0.006^{*}$ & $67.2 \pm 4.8$ & $63.2 \pm 6.5$ & $p=0.003^{3}$ \\
\hline L2-L3 & $54.9 \pm 3.5$ & $51.8 \pm 3.7$ & $p=0.000^{*}$ & $69.5 \pm 5.0$ & $65.4 \pm 5.9$ & $p=0.002^{2}$ \\
\hline L3-L4 & $50.8 \pm 4.3$ & $47.7 \pm 5.9$ & $p=0.012^{*}$ & $68.1 \pm 4.3$ & $62.8 \pm 6.3$ & $p=0.000^{\circ}$ \\
\hline L4-L5 & $43.0 \pm 5.2$ & $39.4 \pm 6.9$ & $p=0.015^{*}$ & $61.5 \pm 5.2$ & $57.9 \pm 5.9$ & $p=0.006$ \\
\hline L5-S1 & $32.8 \pm 6.4$ & $31.1 \pm 5.8$ & $p=0.234$ & $60.3 \pm 7.2$ & $58.7 \pm 7.2$ & $p=0.339$ \\
\hline \multirow{2}{*}{ Level } & \multicolumn{3}{|c|}{ ILFV $\left(\mathrm{mm}^{2}\right)$} & \multicolumn{3}{|c|}{ IFLD (mm) } \\
\hline & Middle & Old & $\mathrm{p}$-value & Middle & Old & $\mathrm{p}$-value \\
\hline L1-L2 & $2195 \pm 329$ & $1772 \pm 394$ & $p=0.000^{*}$ & $23.5 \pm 3.3$ & $18.8 \pm 3.6$ & $p=0.000^{\circ}$ \\
\hline L2-L3 & $2439 \pm 344$ & $1901 \pm 417$ & $p=0.000^{*}$ & $25.0 \pm 4.5$ & $18.8 \pm 3.7$ & $p=0.000^{\circ}$ \\
\hline L3-L4 & $2608 \pm 375$ & $1956 \pm 452$ & $\mathrm{p}=0.000^{*}$ & $22.2 \pm 3.4$ & $17.0 \pm 3.6$ & $p=0.000$ \\
\hline L4-L5 & $2823 \pm 452$ & $2309 \pm 510$ & $p=0.000^{*}$ & $16.4 \pm 3.3$ & $12.8 \pm 3.2$ & $p=0.000^{\circ}$ \\
\hline L5-S1 & $3256 \pm 509$ & $2816 \pm 511$ & $p=0.000^{*}$ & $11.2 \pm 2.3$ & $8.7 \pm 1.9$ & $p=0.000^{*}$ \\
\hline
\end{tabular}

ILFA: inner ligamentum flavum angle, ILFD: inner ligamentum flavum distance, ILEF: inner ligamentum flavum volume, IFLD: inner foraminal ligament distance. Middle: ages 30-49 years; Old: ages 60-79 years. Values are presented as the mean \pm standard deviation.

*Statistically significant difference, $\mathrm{p}<0.05$.
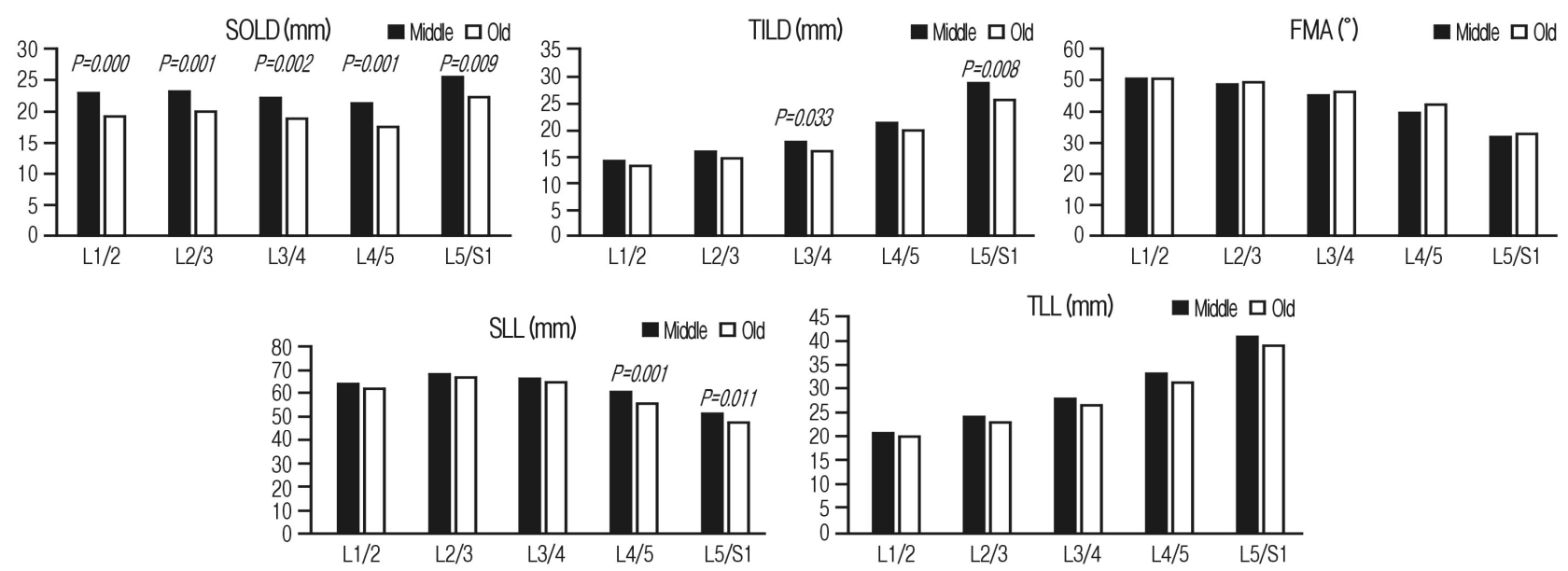

Figure 4. Comparison of outer lamina and outer ligamentum flavum parameters between two age groups. SILD: sagittal interlaminar distance, TILD: Transverse interlaminar distance, FMA: Faceto-midline angle, SLL: sagittal laminae length, TLL: Transverse laminae length. Middle: ages 30-49 years; Old: ages 60-79 years. $p$-value was described in the case of significant difference, $p<0.05$.

ficant differences at all levels (Table 3) (Figure 4). The significant differences were found in the ILFA at the L1-L2, L2-L3, L3-L4, and L4-L5 level $(\mathrm{p}=0.006, \mathrm{p}=0.000, \mathrm{p}=0.012, \mathrm{p}=0.015$, respectively); in the ILFD, at the L1-L2, L2-L3, L3-L4, and L4-L5 level $(p=0.006, p=0.000, p=0.012, p=0.015$, respectively). The ILFV and the IFLD were revealed the significant differences at all levels $(p=0.000$ for all levels) (Table 4) (Figure 5).

\section{DISCUSSION}

In the early phase of development of lumbar endoscopic spine surgery, the focus was on removal of soft disc herniation through the working corridor of Kambin's triangle using transforaminal endoscopic approach ${ }^{3}$. With the introduction of the interlaminar approach, the range of conditions that can be treated by endoscopic spine surgery has expanded with exponential development of surgical equipment and techniques, ${ }^{6,8,15)}$.

Endoscopic surgeons often work in well-defined anatomical corridors that provide a safe working zone for equipment to pass through to achieve targeted decompression and/or fusion. However, the endoscope viewing point is just above the target lesion within the working cannula; the defined anatomical corridor is limited by the working cannula. The endoscopic surgeons should have a lot of experience of other minimally invasive surgery or open surgery for sufficient surgical anatomical config- 

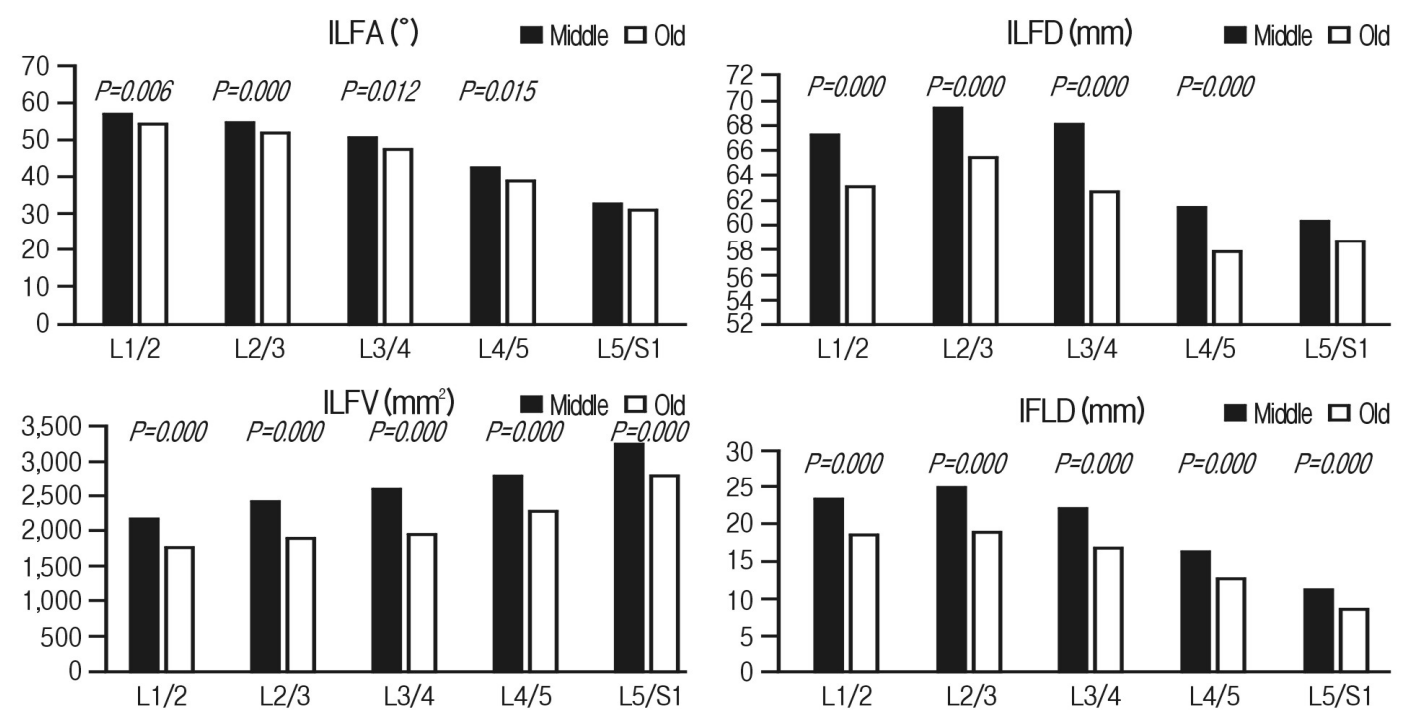

Figure 5. Comparison of inner ligamentum flavum parameters between two age groups. ILFA: inner ligamentum flavum angle, ILFD: inner ligamentum flavum distance, ILFV: inner ligamentum flavum volume, IFLD: inner foraminal ligament distance. Middle: ages 30-49 years; Old: ages 60-79 years. P-value was described in the case of significant difference, $p<0.05$.

uration to complete the targeted decompression within the limited surgical view.

Meanwhile, there was a paucity of literature reporting the surgical anatomical feature, which is essential for interlaminar endoscopic lumbar decompression surgery. The measured parameters should be accurate as numeric values and predictable through the preoperative image like an X-ray and 3D lumbar spine CT to efficiently use anatomical knowledge in endoscopic surgery.

In this context, we decided on the inner LF and the interlaminar window to the critical anatomical structures for interlaminar endoscopic lumbar decompression (IELD) surgery. Because the inner LF is widely distributed from the foramen to the spinal canal's dorsal surface and is a crucial structure that directly compresses neural structure, it could be an anatomical landmark for sufficient decompression surgery (Figure 1). The interlaminar window is the endoscopic entrance to the spinal canal, and its contour directly influences the endoscopic docking site and approach angle.

The inner LF and interlaminar window have changing features according to each lumbar level and age (Figure 1-2). These anatomical changes may differ from the features in the patient who have symptomatic stenosis or disc herniation. Therefore, we included the patient diagnosed with lumbar spinal stenosis and lumbar disc herniation after lumbar MRI and CT image for practical application of results to the IELD operation.

The SILD, TILD, and FMA represent the height, width, and shape of the interlaminar window, respectively. As the disk height decreased with aging, the height of the interlaminar space (SILD) also reduced. As the facet joint arthropathy progressed, hypertrophied capsule and syndesmophytes grew into the lateral angle of the interlaminar window; accordingly, the width (TILD) was getting narrow, and lateral corner became dull, and the angle (FMA) had higher values.

When we performed the IELD surgery, the working cannula is initially docked on the junction of the ipsilateral facet joint and intervertebral disc line on intraoperative fluoroscopy ${ }^{7}$. Soft tissue dissection was done to expose the facet joint medial border and outer LF in the interlaminar window. The drilling is then performed from the medial facet joint border to the medial pedicle line for sufficient traversing root decompression ${ }^{7)}$. In the old age group, as the result of facet arthropathy, the inferior lateral angle of the interlaminar window was occupied with ingrown syndesmophytes and hypertrophied ligaments, so the higher FMA may be measured (Table 3). In these cases, more extent of bone drilling is necessary than the middle age group for sufficient lateral recess decompression (Figure 6-A). Measured SILD, TILD, and FMA results may not be used to an operation as index values due to the anatomical variation of each person, but it could be used effectively to estimate the bone removal extent surrounding the interlaminar window. For example, the FMA values may be more helpful to estimate the bone drilling extent of the medial facet joint part because this value was showed the consistent changing features according to the levels and age groups. Furthermore, we could figure out the shape and approximate angle of the inferior lateral border of the interlaminar window under direct endoscopic vision as expected on the preoperative images.

$S L L$ and TLL values indicate the length and width of the lamina. As the level decreased, SLL values decreased, and TLL values increased, consistent with the well-known lumbar anatomy. The SLL represents the gross anatomical sagittal laminae length on the 3D lumbar CT, which could be changed by the age and degenerative process. As disk height reduction progressed, the 

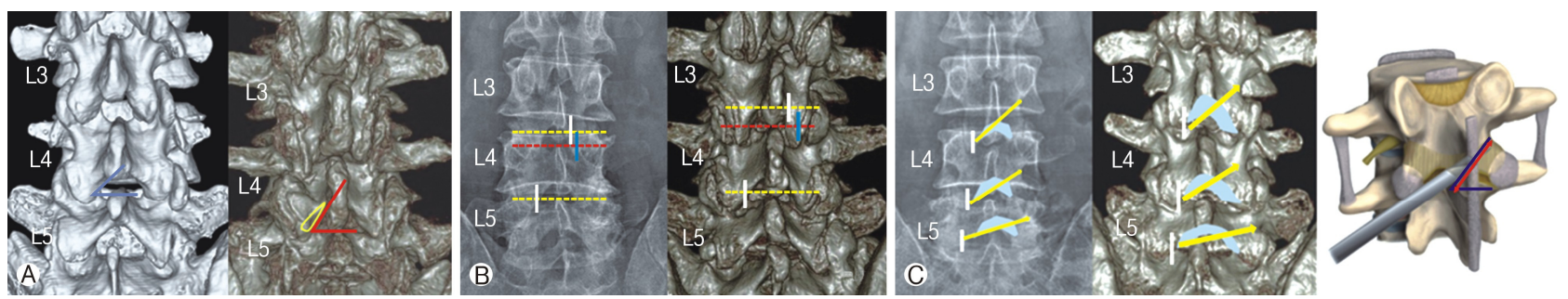

Figure 6. Surgical applications of the measured parameters on the X-ray and 3D CT images. (A): The faceto-midline angle at the L4-L5 level in the middle age group (left, blue line) and old age group (right, red line). The higher angle is shown in the old age group due to the hypertrophied facet joint with the inferior migrated inferior articular process (IAP). More extent of bone drilling is necessary (yellow lined zone) for sufficient lateral recess decompression. (B): Docking point for uniportal interlaminar endoscopic lumbar decompression on the X-ray (left) and 3D lumbar CT (right). The endoscope was initially docked on the junction of the ipsilateral facet joint and intervertebral disc line (yellow dotted line) on intraoperative fluoroscopy (white vertical line). In the IAP inferiorly migrated level, the docking point would be moved more caudally (blue line) base on the superior upper borderline of the lower-level lamina (red dotted line). (C): Application of inner ligamentum flavum angle (ILFA) for interlaminar contralateral endoscopic lumbar foraminotomy (ICELF) on the X-ray (left), 3D lumbar CT (center), and an illustration. The docking point (white vertical line) and the extent of bone drilling (blue color zone) for ICELF are showed on the X-ray and 3D CT. The approach route and angle for the contralateral foramen are described with a yellow arrow line, and the angle gradually decreased as the levels down.

IAP of the upper level moved caudal direction, which then induces the thickening of the LF and narrowing of the interlaminar window. The significant SLL decrease was found at the L4-L5 and L5-S1 levels in the old-age group.

During the operation of the patients with significantly deformed interlaminar window, we would be encountered unusual structures on the limited endoscopic view, and difficult to determine the surgical steps and find the anatomical landmarks. Therefore, the modification of endoscopic docking site for inside canal entrance is necessary to find anatomical landmarks more easily and successful decompression. We recommend moving the endoscopic initial docking site depending on two notable found results in this study rather than the area on the junction of the ipsilateral facet joint and intervertebral disc line.

First, severe facet joint hypertrophy causes the medial facet joint line to move in the medial direction, and this change was documented as the decreased TILD, SILD, and increased FMA. If the docking site is made on the medial facet joint line which have moved medially, the endoscope movement could be limited by narrow space between the spinous process and facet joint. Hence, the vertical line for endoscopic docking is appropriate with mid-point between the medial facet joint line and the medical pedicle line (Figure 6-B)

Second, severely collapsed disc space induces caudal displacement of the upper-level IAP and lamina, and displaced lamina overlaps the interlaminar window documented as a decreased SLL. The Intervertebral disc line position relative to the interlaminar window is moved higher than the disc line position in the normal anatomy. If the endoscopic docking is done on the intervertebral disc line, the first encountered structure may be the spinolaminar part of the upper-level lamina than the interlaminar window or medial facet joint zone. More unnecessary laminotomy is needed to reach the upper and contralateral border of inner LF with this approaching route due to the cranial to caudal approach angle. Therefore, we recommend the upper borderline of the lower-level lamina as a transverse line for docking than the intervertebral disc line to keep the caudal to cranial approach route (Figure 6-B). The caudal to the cranial surgical route during canal decompression is essential to minimize the laminotomy and remove the inner LF with the sublaminar approach.

Contralateral foraminal pathologies could be resolved from the ipsilateral interlaminar endoscopic approach. Recently, Kim et al. published three articles describing the interlaminar contralateral approach for resolving contralateral lateral recess, foraminal, and extraforaminal lesions with technical developments ${ }^{4,5,14)}$. However, the interlaminar endoscopic lumbar foraminotomy (ICELF) procedure is high skill demanding to complete the surgery safely and efficiently because target-oriented endoscopic entry and advancement is essential to reach the foraminal lesion under limited endoscopic view in the narrow sublaminar space $^{14)}$. Although the position of an endoscope could be confirmed with a portable $\mathrm{X}$-ray during operation, initial lesion targeting with proper approaching angle is more important. It is hard to change the endoscopic route during operation due to the surrounding rigid bony structures. In the case of the working cannula have reached to the contralateral foraminal entrance with higher approach angle than the measured value, the advance to extraforaminal part is very difficult, may be impossible without large facet joint resection ${ }^{14)}$. The ILFA represents the endoscopic approach angle from the midline to the upper end of the contralateral foramen, and level and age-related values could be helpful to ICELF procedures (Figure 6-C). The ILFA was revealed the marked low values at the L4-L5 and L5-S1 levels, even lower angle in old age group (Table 4). Hence, nearly horizontal approach angle is used during the ICELF at the L4-L5 and L5-S1 level in old age group.

Aging of the lumbar spine typically manifests as disk height loss and facet joint hypertrophy. The changes of all the measured parameters were closely correlated with these degenerative processes and lead to a reduction of the interlaminar window and distributed area of LF. As a result, the LF is folding into the 
spinal canal, ultimately causing lumbar spinal stenosis, and it was documented as decreased ILFV in the old age group.

Endoscopic spine surgery is performed under a limited surgical view, so predicting essential anatomical changes as described in the present study is crucial for successful decompression surgery. Our research found these changing features on the 3D lumbar CT rather than MRI for practical application to endoscopic lumbar decompression surgery. Furthermore, we used them in the uniportal endoscopic docking site, estimating the bone drilling extent at the medial facet joint part, and approach angle decision for ICELF procedures.

This study had certain limitations. First, this was a single-center retrospective study. Therefore, we could not exclude selection bias. Secondly, the sample of patients may not have represented a diverse population comprising various races or nationalities. Thirdly, the included patients have lumbar spinal stenosis or disc herniation to reinforce clinical application on surgery. Fourthly, we could not determine the exact upper-end point of LF, so we set it at all levels at $8 \mathrm{~mm}$ above the imaginary line that meets the bilateral spinolaminar junctions. Finally, the measured values may not be applied to the surgery directly because those are not fixed absolute values for diverse populations. Nonetheless, this study is important as it is the first attempt to investigate the inner LF contour and interlaminar window boundaries in gross anatomy for endoscopic lumbar spinal surgery. A multicenter study with larger sample size and an improved design would be required to further support the findings of this study.

\section{CONCLUSIONS}

Inner LF distribution and interlaminar window contour are critical anatomical structures for the interlaminar endoscopic lumbar decompression surgery. We found the changing features of these structures correlated with age and levels to help with endoscopic decompression surgery has a limited surgical corridor. The present study results may help the endoscopic surgeons decide the endoscopic docking site, the extent of bone drilling at the medial facet joint part, and the approach angle for contralateral lumbar foraminotomy.

\section{CONFLICT OF INTEREST}

Dr. HS Kim is an editorial board member of the journal but was not involved in the peer reviewer selection, evaluation, or decision process of this article. There are no other potential conflicts of interest relevant to this article to declare.

\section{REFERENCES}

1. Boissiere L, Moal B, Gille O, De-Roquefeuil E, Durieux M, Obeid I, et al.: Lumbar spinal muscles and spinal canal study by MRI three-dimensional reconstruction in adult lumbar spinal stenosis. Orthop Traumatol Surg Res 103:279-283, 2017

2. Chau AM, Pelzer NR, Hampton J, Smith A, Seex KA, Stewart F, et al.: Lateral extent and ventral laminar attachments of the lumbar ligamentum flavum: Cadaveric study. Spine J 14:24672471, 2014

3. Kambin P, Sampson S: Posterolateral percutaneous suction-excision of herniated lumbar intervertebral discs. Report of interim results. Clin Orthop Relat Res: 37-43, 1986

4. Kim HS, Patel R, Paudel B, Jang JS, Jang IT, Oh SH, et al.: Early Outcomes of Endoscopic Contralateral Foraminal and Lateral Recess Decompression via an Interlaminar Approach in Patients with Unilateral Radiculopathy from Unilateral Foraminal Stenosis. World Neurosurg 108:763-773, 2017

5. Kim HS, Singh R, Adsul NM, Oh SW, Noh JH, Jang IT: Management of Root-Level Double Crush: Case Report with Technical Notes on Contralateral Interlaminar Foraminotomy with Full Endoscopic Uniportal Approach. World Neurosurg 122:505-507, 2019

6. Kim HS, Wu PH, Jang IT: Development of Endoscopic Spine Surgery for Healthy Life: To Provide Spine Care for Better, for Worse, for Richer, for Poorer, in Sickness and in Health. Neurospine 17:S3-S8, 2020

7. Kim HS, Wu PH, Jang IT: Lumbar Endoscopic Unilateral Laminotomy for Bilateral Decompression Outside-In Approach: A Proctorship Guideline With 12 Steps of Effectiveness and Safety. Neurospine 17:S99-S109, 2020

8. Kim M, Kim HS, Oh SW, Adsul NM, Singh R, Kashlan ON, et al.: Evolution of Spinal Endoscopic Surgery. Neurospine 16: 6-14, 2019

9. Losiniecki AJ, Serrone JC, Keller JT, Bohinski RJ: Lumbar ligamentum flavum: spatial relationships to surrounding anatomical structures and technical description of en bloc resection. J Neurol Surg A Cent Eur Neurosurg 74:388-392, 2013

10. Olszewski AD, Yaszemski MJ, White AA, $3^{\text {rd }}$ : The anatomy of the human lumbar ligamentum flavum. New observations and their surgical importance. Spine (Phila Pa 1976) 21:2307-2312, 1996

11. Reyes-Sanchez A, Garcia-Ramos CL, Deras-Barrientos CM, Alpizar-Aguirre A, Rosales-Olivarez LM, Pichardo-Bahena R: Ligamentum flavum in lumbar spinal stenosis, disc herniation and degenerative spondylolisthesis. An histopathological description. Acta Ortop Mex 33:308-313, 2019

12. Safak AA, Is M, Sevinc O, Barut C, Eryoruk N, Erdogmus B, et al.: The thickness of the ligamentum flavum in relation to age and gender. Clin Anat 23:79-83, 2010

13. Winkler PA, Zausinger S, Milz S, Buettner A, Wiesmann M, Tonn JC: Morphometric studies of the ligamentum flavum: A correlative microanatomical and MRI study of the lumbar spine. Zentralbl Neurochir 68:200-204, 2007

14. Wu PH, Kim HS, Jang IT: How I do it? Uniportal full endoscopic contralateral approach for lumbar foraminal stenosis with double crush syndrome. Acta Neurochir (Wien) 162:305-310, 2020

15. Wu PH, Kim HS, Jang IT: A Narrative Review of Development of Full-Endoscopic Lumbar Spine Surgery. Neurospine 17:S20S33, 2020 